\title{
Endovascular Treatment of Posterior Communicating Artery Aneurysms with Oculomotor Nerve Palsy: Clinical Outcomes and Predictors of Nerve Recovery
}

\author{
N. Chalouhi, T. Theofanis, P. Jabbour, A.S. Dumont, L.F. Gonzalez, R.M. Starke, D. Gordon, R. Rosenwasser, and S. Tjoumakaris
}

\begin{abstract}
BACKGROUND AND PURPOSE: ONP is a well-known presentation of PcomA aneurysms. Reports on recovery of ONP with endovascular coiling have been limited to small case series. We assessed the safety and efficacy of endovascular therapy in a series of PcomA aneurysms with ONP.
\end{abstract}

MATERIALS AND METHODS: We reviewed 37 patients with ONP who underwent endovascular treatment in our institution between 2005 and 2011. Published studies were also reviewed to determine the overall rate of ONP recovery with endovascular therapy.

RESULTS: Nineteen patients (51.4\%) presented with complete ONP, and 18 (48.6\%), with partial ONP. Conventional coiling was performed in 31 (83.8\%) patients; stent-assisted coiling, in 4 (10.8\%); and balloon remodeling, in 2 (5.4\%). There was 1 (2.7\%) procedural complication (a transient thromboembolic event). Twenty-seven (73\%) patients were treated within 3 days from symptom onset. At the last available clinical follow-up, ONP resolution was complete in 14 (37.8\%) patients and partial in 19 (51.4\%). Only 4 (10.8\%) patients showed no signs of nerve recovery. In multivariate analysis, partial ONP and longer follow-up durations were predictors of complete nerve recovery. Treatment timing, type of endovascular embolization, subarachnoid hemorrhage, and initial degree of aneurysm occlusion were not predictors of nerve recovery. Of 169 patients reported in the literature (including ours), ONP resolved completely in 73 (43.2\%) patients and partially in 73 (43.2\%).

CONCLUSIONS: Endovascular therapy is a safe and highly efficient alternative to surgical clipping for PcomA aneurysms with ONP.

ABBREVIATIONS: ISAT = International Subarachnoid Aneurysm Trial; ONP = oculomotor nerve palsy; OR = odds ratio; PcomA = posterior communicating artery

O NP is a well-known presentation of both ruptured and unruptured PcomA aneurysms. ${ }^{1-3}$ Treatment is indicated to prevent the imminent risk of aneurysm rupture or rerupture and to hasten nerve recovery. The optimal management strategy of these lesions remains largely unknown. Direct compression of the oculomotor nerve by the aneurysmal mass is thought to be the main mechanism of nerve injury. Accordingly, surgical clipping has been regarded as the preferred treatment technique because it allows immediate alleviation of the mass effect. ${ }^{4,5}$ Endovascular therapy has

Received May 14, 2012; accepted after revision June 25.

From the Department of Neurosurgery (N.C., T.T., P.J., A.S.D., L.F.G., D.G., R.R., S.T.), Thomas Jefferson University and Jefferson Hospital for Neuroscience, Philadelphia, Pennsylvania; and Department of Neurological Surgery (R.M.S.), University of Virginia School of Medicine, Charlottesville, Virginia.

Abstract previously presented as an oral presentation (neurosurgical forum) at: Annual Meeting of the Congress of Neurological Surgeons, October 6-12, 2012; Chicago, Illinois.

Please address correspondence to Stavropoula I. Tjoumakaris, MD, Department of Neurological Surgery, Division of Neurovascular Surgery and Endovascular Neurosurgery, Thomas Jefferson University Hospital, 901 Walnut St, 3rd Floor, Philadelphia, PA 19107; e-mail: stavropoula.tjoumakaris@jefferson.edu

http://dx.doi.org/10.3174/ajnr.A3294 been cautiously used to treat PcomA aneurysms with ONP because of concerns that coiling could contribute to the mass effect and compromise nerve recovery. The rates of oculomotor nerve recovery with microsurgical and endovascular treatment have varied widely in published series. ${ }^{1,4-7}$ A meta-analysis suggested that surgical treatment may be superior to endovascular embolization with respect to ONP resolution. ${ }^{5}$ Recently published studies have reported remarkably high rates of complete nerve recovery with endovascular therapy (50\%-85\%), equaling or even exceeding those observed with surgical clipping. ${ }^{8-10}$ Results of these studies, however, are limited by the sample size, which precludes any confident conclusion as to the efficacy of endovascular therapy in this setting.

We investigated the clinical outcome of ONP with different endovascular embolization techniques in a series of 37 patients treated in our institution. A multivariate logistic regression analysis was performed to determine the predictors of oculomotor nerve recovery after endovascular treatment. We also systematically reviewed the literature and combined our results with those of previously published series to determine the overall rate of ONP resolution with endovascular therapy. 


\section{MATERIALS AND METHODS}

The institutional review board approved the study protocol. Between 2006 and 2011, 356 patients with PcomA aneurysms underwent endovascular treatment at our institution. Of the 356 patients, $42(11.8 \%)$ presented with ONP. Thirty-seven of these 42 patients $(88.1 \%)$ had at least 1 documented follow-up visit and constituted our study population. Medical charts, operative reports, and office follow-up records were carefully reviewed to determine the patient age, sex, size of aneurysm, Hunt and Hess grades, degree of ONP, time from symptom onset to treatment, type of endovascular technique, procedural complications, immediate angiographic results, and extent of nerve recovery after endovascular therapy.

ONP was considered complete in the presence of the following: 1) ptosis; 2) fixed mydriasis; 3) diplopia; and 4) complete upward, medial, and downward-gaze palsies. ONP was defined as partial if any of these signs and symptoms were absent. Careful review of patient radiographic and clinical data excluded mydriasis as a result of uncal herniation. Follow-up visits were scheduled at 3, 6, and 12 months after the initial intervention and annually thereafter. Complete ONP recovery was defined as complete resolution of the patient's initial signs and symptoms except for subtle ptosis and slight differences in pupillary reactions. Partial ONP recovery was defined as residual diplopia/ ophthalmoplegia, residual ptosis, or residual pupillary dysfunction.

Patients presenting with subarachnoid hemorrhage or acute ONP were typically treated within 24 hours of admission. Only bare platinum coils were used for aneurysm embolization. Coils were placed until satisfactory aneurysm obliteration was achieved. The degree of aneurysm occlusion was determined by the operator at the time of the procedure. Aneurysms with wide necks or unfavorable neck-to-dome ratios were coiled by using stent or balloon assistance.

\section{Statistical Analysis}

Data are presented as mean and range for continuous variables and as frequency for categoric variables. Statistical analyses were performed by using an unpaired Student $t$ test, $\chi^{2}$ test, and Fisher exact test, as appropriate. Odds ratios were calculated by using univariate logistic regression analysis. Factors predictive of nerve recovery in univariate analysis $(P<.15)$ were entered into a stepwise backwards multivariate logistic regression analysis. $P$ values of $\leq .05$ were considered statistically significant.

\section{Literature Search}

Published studies on the recovery of ONP with endovascular therapy were identified by using a Medline search with a combination of the following key words: "posterior communicating artery aneurysms," "ophthalmoplegia," "third nerve palsy," "oculomotor nerve palsy," and "endovascular." Additional articles were obtained by cross-referencing from relevant reviews and original articles. Case reports and series involving $<3$ patients were excluded from the analysis. Valid studies were reviewed to determine the outcome of patients with ONP undergoing endovascular treatment. ONP recovery was categorized as "complete recovery," "partial recovery," and "no recovery."

\section{RESULTS}

\section{Demographics and Aneurysm Characteristics}

Of the 37 patients included in the study, 29 (78.4\%) were women and $8(21.6 \%)$ were men. The mean age in the series was 54.8 years (range, 32-84 years). Hunt and Hess grades were 0 in 20 (54.1\%) patients, I in $9(24.3 \%)$ patients, II in $3(8.1 \%)$ patients, III in 3 $(8.1 \%)$ patients, and IV in $2(5.4 \%)$ patients. Mean aneurysm size was $7.6 \mathrm{~mm}$ (range, $4-13 \mathrm{~mm}$ ) in the present series. There were 6 (16.2\%) aneurysms larger than $10 \mathrm{~mm}, 30(81.1 \%)$ aneurysms between 5 and $10 \mathrm{~mm}$, and only 1 (2.7\%) aneurysm smaller than $5 \mathrm{~mm}$. Aneurysm size was significantly smaller in the cohort of patients with PcomA aneurysms who did not present with ONP (6.4 $\mathrm{mm}, n=314, P=.02$ )

\section{Endovascular Treatment}

Endovascular treatment was successful in all 37 patients (100\%). Conventional coiling was performed in 31 (83.8\%) patients; stent-assisted coiling, in $4(10.8 \%)$; and balloon remodeling, in 2 (5.4\%). The Neuroform stent (Boston Scientific, Natick, Massachusetts) was used in 2 patients; the Enterprise stent (Cordis Neurovascular, Miami Lakes, Florida), in 2; and the HyperGlide balloon (ev3, Irvine, California) in 2. The interval between the onset of ONP and endovascular treatment was $0-3$ days in $27(73 \%)$ patients, $4-14$ days in $5(13.5 \%)$ patients, and $>14$ days in 5 (13.5\%) patients. Complete aneurysm occlusion (100\%) was achieved in 19 (51.4\%) patients; near-complete occlusion (95\%$99 \%)$, in $14(37.8 \%)$; and incomplete occlusion $(<95 \%)$, in 4 (10.8\%). Only $1(2.7 \%)$ patient experienced a procedural complication. This was a 61-year-old patient with a grade IV subarachnoid hemorrhage who developed a thrombus occluding the M2 segment of the middle cerebral artery intraoperatively. Chemical and mechanical thrombolysis failed to recanalize the occluded artery before it was successfully reopened with stent angioplasty (Enterprise). An infarct developed postoperatively in the middle cerebral artery territory but left no permanent sequelae. There were no deaths or permanent morbidity related to the procedure. No early or late hemorrhage was observed after endovascular aneurysm treatment in the series.

\section{ONP Palsy and Its Recovery}

Nineteen (51.4\%) patients presented with complete ONP; and 18 (48.6\%), with partial ONP. Mean follow-up time was 11.3 months (range, 3-48 months). At the last available clinical follow-up, ONP resolution was complete in $14(37.8 \%)$ patients and partial in $19(51.4 \%)$ patients. Only $4(10.8 \%)$ patients showed no signs of oculomotor nerve recovery; 2 of these patients had a complete ONP and 2 had a partial ONP. Complete nerve recovery occurred in 50\% (9/18) of patients with initial partial ONP versus $21 \%(4 / 19, P=.01)$ with initial complete ONP. ONP recovery occurred mostly within the first year following treatment. However, we are aware of at least 2 patients who recovered in a delayed fashion, namely after their 1-year clinical follow-up.

\section{Predictors of Complete ONP Recovery}

Factors predictive of complete ONP recovery in univariate analysis were complete aneurysm occlusion, partial ONP palsy, and increasing follow-up time. Patient sex and age, aneurysm size, 


\begin{tabular}{|c|c|c|c|c|}
\hline & No. of Patients & Complete Recovery (\%) & Partial Recovery (\%) & No Recovery (\%) \\
\hline Birchall et al ${ }^{20}(1999)$ & 3 & $3(100)$ & 0 & 0 \\
\hline Stiebel-Kalish et al $^{\prime}$ (2003) & 11 & 0 & $3(27.3)$ & $8(72.7)$ \\
\hline Kim et $\mathrm{al}^{21}(2003)$ & 3 & 0 & $3(100)$ & 0 \\
\hline Chen et $\mathrm{al}^{4}(2006)$ & 6 & $2(33.3)$ & $3(50)$ & $1(16.7)$ \\
\hline Ahn et $\mathrm{al}^{14}(2006)$ & 10 & $6(60)$ & $3(30)$ & $1(10)$ \\
\hline Mansour et al $\mathrm{l}^{10}(2007)$ & 7 & $6(85.7)$ & 0 & $1(14.3)$ \\
\hline Hanse et $\mathrm{al}^{11}(2008)$ & 21 & $8(38.1)$ & $11(52.4)$ & $2(9.5)$ \\
\hline Güresir et $\mathrm{al}^{5}$ (2011) & 7 & $3(42.9)$ & $4(57.1)^{\prime}$ & 0 \\
\hline Zhang et $\mathrm{al}^{8}$ (2011) & 13 & $7(53.8)$ & $6(46.2)$ & 0 \\
\hline Kassis et $\mathrm{al}^{6}(2010)$ & 20 & $7(35)$ & $12(60)$ & $1(5)$ \\
\hline Santillan et $\mathrm{al}^{12}(2010)$ & 11 & $7(63.6)$ & $2(18.2)$ & $2(18.2)$ \\
\hline Ko and $\mathrm{Kim}^{9}$ (2011) & 10 & $7(70)$ & $1(10)$ & $2(20)$ \\
\hline Panagiotopoulos et $\mathrm{al}^{22}$ (2011) & 10 & $3(30)$ & $6(60)$ & $1(10)$ \\
\hline Present series (2012) & 37 & $14(37.8)$ & $19(51.4)$ & $4(10.8)$ \\
\hline Total & 169 & 73 (43.2) & $73(43.2)$ & $23(13.6)$ \\
\hline
\end{tabular}

ruptured/unruptured aneurysm status, Hunt and Hess grades, type of endovascular technique, and time from symptom onset to treatment were not shown to be predictive factors. Predictive factors were subsequently entered into a multivariate logistic regression analysis. At the significance level of .05, a partial ONP palsy was the only predictor of complete $\mathrm{ONP}$ recovery $(\mathrm{OR}=8.06$; $P=.02)$. There was also a trend toward complete ONP recovery with increasing follow-up time $(\mathrm{OR}=1.2 ; P=.081)$.

\section{Predictors of ONP Recovery}

A second multivariate analysis was performed to identify predictors of ONP recovery to any degree (partial or complete). None of the previously cited factors were found to be a statistically significant predictor of ONP recovery.

\section{Published Data}

The results of previously published series on the recovery of ONP with endovascular treatment are summarized in the Table. To date, a total of 169 patients with PcomA aneurysms causing ONP (including our series) underwent endovascular treatment. Overall, ONP resolved completely in $73(43.2 \%)$ patients and partially in $73(43.2 \%)$ patients, while $23(13.6 \%)$ patients showed no signs of recovery.

\section{DISCUSSION}

The best treatment strategy for PcomA aneurysms with ONP remains unknown. Some authors recommend surgical clipping to alleviate mass effect and allow prompt nerve recovery. ${ }^{4,5}$ Endovascular therapy has also shown high efficacy in promoting nerve recovery. ${ }^{9,11,12}$ Experience with endovascular therapy, however, has been limited to a few case series of small sample size. We assessed the resolution of ONP in a series of 37 patients undergoing endovascular treatment in our institution. We found that endovascular therapy allowed complete nerve recovery in $37.8 \%$ of patients and partial recovery in $51.4 \%$, while only $10.8 \%$ remained unchanged. Therefore, overall improvement (partial or complete) occurs in up to $90 \%$ of patients undergoing endovascular therapy. These important findings are in agreement with the findings of Hanse et $\mathrm{al},{ }^{11}$ who assessed the recovery of ONP with endovascular therapy in 21 patients. The authors found that ONP recovered completely in $38.1 \%(8 / 21)$ of patients and partially in
$52.4 \%(11 / 21)$ after endovascular therapy. In another study that included 20 patients, Kassis et $\mathrm{al}^{6}$ reported complete ONP recovery in $35 \%(7 / 20)$ of cases and partial recovery in $60 \%(12 / 20)$. Smaller studies have reported higher recovery rates with endovascular therapy, but these studies are particularly prone to sampling errors. In a series of 10 patients undergoing endovascular coiling, Ko and $\mathrm{Kim}^{9}$ noted complete recovery in 7 patients $(70 \%)$ and partial recovery in 1 patient (10\%). In another small endovascular series that included 11 patients, Santillan et al ${ }^{12}$ reported complete resolution of ONP in 7 patients $(63.6 \%)$ and partial resolution in 2 patients $(18.2 \%)$. When the results of all endovascular series including ours were combined, both complete and partial ONP recovery was found to occur in $43.2 \%$ of cases. On the basis of these findings, endovascular therapy appears to be highly efficient in promoting oculomotor nerve recovery in patients with PcomA aneurysms. The exact mechanism by which coiling promotes oculomotor nerve recovery is not well understood. A commonly proposed explanation for this finding is that coiling removes aneurysmal pulsations, thereby allowing more complete nerve recovery. ${ }^{4,13}$

Few studies have compared the efficacy of surgical and endovascular treatment in patients with ONP. In a retrospective study comparing the outcomes of ONP in 13 patients, Chen et $\mathrm{al}^{4}$ reported complete recovery in 6 of 7 patients undergoing surgical clipping versus only 2 of 6 patients undergoing endovascular coiling. The authors concluded that clipping is superior to coiling and should be considered the treatment of choice in patients who can tolerate surgery. In another small retrospective study, Güresir et $\mathrm{al}^{5}$ reported higher recovery rates after surgical clipping, with complete ONP resolution occurring in 4 of 4 surgical patients versus only 3 of 7 endovascular patients. However, given the small sample size and the high probability of a type I error, we believe that no firm conclusions can be drawn from these reports regarding the optimal treatment strategy for PcomA aneurysms with ONP. Evidence from large prospective studies is clearly necessary to establish the superiority of one technique over another. In their systematic review of the literature regarding ONP related to PcomA aneurysms, Güresir et al found that partial and complete ONP recovery occurred, respectively, in 38\% (50/132) and 55\% (72/132) of patients undergoing surgical clipping versus $43 \%$ (23/ $54)$ and $32 \%(17 / 54)$ of those undergoing endovascular emboli- 
zation. Surgical clipping was found to be a significant predictor of ONP recovery in multivariate analysis. However, this report has inherent limitations because it combines data from small retrospective series, with the potential of pooling biases and limitations of individual studies, into 1 analysis. In a well-designed study, Ahn et $\mathrm{al}^{14}$ compared 10 patients with ONP treated with endovascular coiling and 7 patients treated with surgical clipping and reported no difference in nerve recovery between the 2 groups. Our systematic review of the literature confirms the efficacy of endovascular therapy because the overall rate of partial or complete nerve recovery was as high as $86.4 \%$.

We were able to perform endovascular embolization of PcomA aneurysms with a rate of procedural complications of $2.7 \%$. No procedure-related permanent morbidity or deaths were noted. We believe that the safety of endovascular therapy in this setting is an equally important factor that should also be taken into account when considering treatment options. Although the main goal of the current study was to specifically assess the outcome of ONP, the minimal invasive approach of endovascular therapy and prompt clinical recovery from surgery should be considered. In fact, the results of the ISAT and recently the Barrow Ruptured Aneurysm Trial have clearly demonstrated that endovascular treatment is associated with an improved functional outcome compared with open surgery. ${ }^{15,16}$ Overall, endovascular therapy appears to have a favorable safety and efficacy profile in patients with PcomA aneurysms presenting with ONP.

The relationship between aneurysm size and the development of ONP is not well understood. It makes intuitive sense that large PcomA aneurysms are more likely to generate mass effect and result in ONP. In the current study, we found that PcomA aneurysms causing ONP were indeed significantly larger than those not causing ONP; this finding suggests that aneurysm size is a decisive factor in the development of ONP. Along these lines, Teasdale et al $^{17}$ suggested that unruptured PcomA aneurysms would need to be at least $7 \mathrm{~mm}$ in diameter to cause ONP. In contrast to their findings, however, ONP developed in 2 patients with unruptured aneurysms measuring only $5 \mathrm{~mm}$ in diameter in the present series. Patient anatomy could account for an occasional ONP occurring with small unruptured PcomA aneurysms.

A variety of factors have been found to influence ONP recovery in the literature, including the degree of initial palsy, ${ }^{4,5,7,8,11,18}$ treatment timing, ${ }^{8,11,19}$ subarachnoid hemorrhage, ${ }^{6,8,10}$ patient age, ${ }^{14}$ and cardiovascular risk factors. ${ }^{14}$ In the present study, a partial ONP at presentation was the only statistically significant predictor of complete ONP recovery with endovascular therapy. As such, ONP resolution was complete in $50 \%$ of patients with partial ONP versus only $21 \%$ in patients with complete ONP. In multivariate analysis, the odds of progression to complete ONP recovery were 8 times more likely for patients with partial ONP after controlling for other variables. These findings are consistent with those of Zhang et $\mathrm{al},{ }^{8}$ who reported a rate of complete nerve recovery as high as $100 \%$ in patients presenting with partial ONP versus only $25 \%$ in those presenting with complete ONP. Likewise, Chen et $\mathrm{al}^{4}$ noted that ONP recovered completely in 5 of 6 patients with partial ONP versus 3 of 7 patients with complete ONP. In fact, in patients with partial palsy, the oculomotor nerve is likely to be in a neuropraxic state and has, therefore, a better chance of full recovery.

The length of follow-up time is another factor that was found to influence complete recovery rates with endovascular therapy in the present study, though it did not reach statistical significance. This finding is potentially crucial because it implies that the odds of making a full recovery after endovascular therapy increase with time. The observation that 2 patients recovered fully in a delayed fashion (after the 1-year follow-up) further supports this statement. On the basis of these findings, it is possible that a significant number of patients with partial ONP recovery at their latest follow-up would eventually progress to complete recovery. Our findings contradict those of Chen et $\mathrm{al}^{4}$ and Hanse et al, ${ }^{11}$ who respectively reported that $\mathrm{ONP}$ recovery occurs invariably within 6 and 12 months following treatment. This question, however, requires further investigation ideally through large prospective studies with long periods of clinical follow-up. Despite being an important factor in ONP development, aneurysm size does not seem to affect the recovery of oculomotor function. In addition, the type of endovascular technique was not a factor in ONP recovery.

Finally, we could not confirm previous reports of higher ONP recovery rates with earlier intervention or in patients with subarachnoid hemorrhage. Leivo et al ${ }^{19}$ surgically treated 28 patients with ONP and reported complete nerve recovery in up to $88 \%$ of patients undergoing surgery within 3 days versus only $30.8 \%$ of those undergoing surgery after more than 6 days. The authors concluded that early surgery (within 3 days) improves the cure of PcomA aneurysm-induced ONP.

The limitations of our study stem primarily from its retrospective design and the lack of a control surgical group. Additionally, the rate of complete ONP recovery could be somewhat underestimated in the present report given the lack of long-term follow-up in some patients. Actually, this would further support the efficacy of endovascular therapy because excellent recovery rates were still achieved. Prospective randomized trials with long periods of follow-up are needed to establish the best treatment strategy for this patient population.

\section{CONCLUSIONS}

In this study, we presented the results of a series of 37 patients undergoing endovascular treatment for PcomA aneurysms with ONP. We found that ONP recovery occurred in nearly $90 \%$ of patients after endovascular treatment and that the degree of initial ONP was the major factor predicting complete nerve recovery. Our findings suggest that endovascular therapy is a safe and highly efficient alternative to surgical clipping for PcomA aneurysms with ONP.

Disclosures: Pascal Jabbour-UNRELATED: Consultancy: ev3, Codman Neurovascular. Aaron S. Dumont-UNRELATED: Consultancy: ev3, Stryker Neurovascular. Robert Rosenwasser-UNRELATED: Grants/Grants Pending: Cordis, NIH, Comments: Cordis: Sapphire WW Post Market Surveillance Study, protocol P06-3603; NIH: Carotid Revascularization Endarterectomy vs Stent Trial; NIH: SAMMPRIS.* Stavropoula Tjoumakaris_UNRELATED: Consultancy: Stryker Endovascular, Comments: Endovascular Fellows Course Instructor. *Money paid to the institution.

\section{REFERENCES}

1. Stiebel-Kalish H, Maimon S, Amsalem J, et al. Evolution of oculomotor nerve paresis after endovascular coiling of posterior communi-

AJNR Am J Neuroradiol 34:828-32 Apr 2013 www.ajnr.org 831 
cating artery aneurysms: a neuro-ophthalmological perspective. Neurosurgery 2003;53:1268-73, discussion 1273-74

2. Soni SR. Aneurysms of the posterior communicating artery and oculomotor paresis. J Neurol Neurosurg Psychiatry 1974;37:475-84

3. Sorimachi T, Fujii Y, Nashimoto T. A true posterior communicating artery aneurysm: variations in the relationship between the posterior communicating artery and the oculomotor nerve-case illustration. J Neurosurg 2004;100:353

4. Chen PR, Amin-Hanjani S, Albuquerque FC, et al. Outcome of oculomotor nerve palsy from posterior communicating artery aneurysms: comparison of clipping and coiling. Neurosurgery 2006; 58:1040-46, discussion 1040-46

5. Güresir E, Schuss P, Setzer M, et al. Posterior communicating artery aneurysm-related oculomotor nerve palsy: influence of surgical and endovascular treatment on recovery: single-center series and systematic review. Neurosurgery 2011;68:1527-33, discussion 1533-34

6. Kassis SZ, Jouanneau E, Tahon FB, et al. Recovery of third nerve palsy after endovascular treatment of posterior communicating artery aneurysms. World Neurosurg 2010;73:11-16, discussion e2

7. Javalkar V, Cardenas R, Nanda A. Recovery of third nerve palsy following surgical clipping of posterior communicating artery aneurysms. World Neurosurg 2010;73:353-56

8. Zhang SH, Pei W, Cai XS, et al. Endovascular management and recovery from oculomotor nerve palsy associated with aneurysms of the posterior communicating artery. World Neurosurg 2010;74: $316-19$

9. Ko JH, Kim YJ. Oculomotor nerve palsy caused by posterior communicating artery aneurysm: evaluation of symptoms after endovascular treatment. Interv Neuroradiol 2011;17:415-19

10. Mansour N, Kamel MH, Kelleher M, et al. Resolution of cranial nerve paresis after endovascular management of cerebral aneurysms. Surg Neurol 2007;68:500-04, discussion 504

11. Hanse MC, Gerrits MC, van Rooij WJ, et al. Recovery of posterior communicating artery aneurysm-induced oculomotor palsy after coiling. AJNR Am J Neuroradiol 2008;29:988-90
12. Santillan A, Zink WE, Knopman J, et al. Early endovascular management of oculomotor nerve palsy associated with posterior communicating artery aneurysms. Interv Neuroradiol 2010;16:17-21

13. Albuquerque FC. Coiling versus clipping for posterior communicating artery aneurysms associated with oculomotor nerve palsy: only time will tell. World Neurosurg 2010;74:250-51

14. Ahn JY, Han IB, Yoon PH, et al. Clipping vs coiling of posterior communicating artery aneurysms with third nerve palsy. Neurology 2006;66:121-23

15. McDougall CG, Spetzler RF, Zabramski JM, et al. The Barrow Ruptured Aneurysm Trial. J Neurosurg 2012;116:135-44

16. Molyneux AJ, Kerr RS, Yu LM, et al. International subarachnoid aneurysm trial (ISAT) of neurosurgical clipping versus endovascular coiling in 2143 patients with ruptured intracranial aneurysms: a randomised comparison of effects on survival, dependency, seizures, rebleeding, subgroups, and aneurysm occlusion. Lancet 2005;366:809-17

17. Teasdale E, Statham P, Straiton J, et al. Non-invasive radiological investigation for oculomotor palsy. J Neurol Neurosurg Psychiatry 1990;53:549-53

18. Giombini S, Ferraresi S, Pluchino F. Reversal of oculomotor disorders after intracranial aneurysm surgery. Acta Neurochir (Wien) 1991;112:19-24

19. Leivo S, Hernesniemi J, Luukkonen M, et al. Early surgery improves the cure of aneurysm-induced oculomotor palsy. Surg Neurol 1996;45:430-34

20. Birchall D, Khangure MS, McAuliffe W. Resolution of third nerve paresis after endovascular management of aneurysms of the posterior communicating artery. AJNR Am J Neuroradiol 1999;20:411-13

21. Kim DJ, Kim DI, Lee SK, et al. Unruptured aneurysms with cranial nerve symptoms: efficacy of endosaccular Guglielmi detachable coil treatment. Korean J Radiol 2003;4:141-45

22. Panagiotopoulos V, Ladd SC, Gizewski E, et al. Recovery of ophthalmoplegia after endovascular treatment of intracranial aneurysms. AJNR Am J Neuroradiol 2011;32:276-82 